\title{
mHealth technologies in the management of obesity: a narrative review
}

This article was published in the following Dove Press journal:

Smart Homecare Technology and TeleHealth

18 July 2017

Number of times this article has been viewed

\section{Margaret Allman-Farinelli Juliana Chen}

School of Life and Environmental Science, Charles Perkins Centre, The University of Sydney, Camperdown, NSW, Australia
Correspondence: Margaret Allman-Farinelli School of Life and Environmental Science, Charles Perkins Centre DI7, The University of Sydney, Camperdown, NSW 2006, Australia

Tel +6I 290367045

Fax +6I $28627 \mid 605$ Email margaret.allmanfarinelli@sydney. edu.au
Abstract: The global pandemic of obesity requires an urgent response. Obesity results in increased risk of type 2 diabetes, cardiovascular disease and cancer. The need to provide costeffective treatment to the millions affected is evident but traditional repeated individual face-toface management would clearly exhaust available health care services. It is for this reason that the use of mobile health (mHealth) to improve population health, diet, and physical activity has become an active area of research. The aim of this review is to give a perspective of where this field of research currently stands using examples from the published literature. Smartphones provide a medium for the delivery of health information, personal coaching, monitoring of eating and physical activity behaviors and to integrate outputs from other sensors. Health and computer researchers have been developing and evaluating mHealth for the management of obesity but their efforts are relatively small. Text messaging, applications (apps) and a number of multicomponent mHealth programs utilizing a variety of Smartphone functions indicate some success in weight management but the results are mixed. Apart from the ubiquitous nature of Smartphones and devices, the real-time delivery of output data provides the potential for continuous adjustment of interventions according to the changes an individual is making after each message they receive from the mHealth program. This ability to optimize interventions for individual weight management in a timely fashion is the big challenge for researchers. Personalized heath advice is the preferred option of individuals, and cooperation between computer scientists, behavioral scientists, dietitians, exercise physiologists and medical practitioners will be needed to meaningfully integrate data and responses from automated systems. In summary, mHealth shows potential, but we are still in the early stages of being able to harness its full capabilities for making a meaningful contribution to combatting obesity.

Keywords: mHealth, text messages, apps, obesity, diet, physical activity

\section{Introduction}

The world is in the midst of an obesity pandemic. With more than 1.9 billion overweight and 600 million obese, the global response is urgent. ${ }^{1}$ Obesity is a major cause of type 2 diabetes, cardiovascular disease and a large number of cancers, such as breast, colorectal, prostate, esophageal, liver and kidney. ${ }^{2}$ The global burden of overweight and obesity is frequently expressed in terms of disability adjusted life-years (DALYs), which is the sum of years of life lost and years of life living with disability, contributed to exposure to the risk factor. Overweight and obesity were found to contribute to a loss of 120.1 million years (95\% confidence interval [CI] 83.8 to 158.4 million). ${ }^{3}$ The number of obese children younger than 5 years old was estimated to be 41 million. ${ }^{1}$ The rates of obesity are generally higher in wealthy developed nations, but the problem 
is increasing in low- and middle-income countries. There are now more people with a high body fat than with malnutrition in the world. ${ }^{3}$ With such a large percentage of the global population needing treatment for their obesity, and many more needing management of their overweight and weight gain to prevent obesity, multiple population-based rather than individual approaches are necessary. Policies aimed at changing food environments and the opportunities for physical activity are important aspects of the solution, as are mass media social marketing and education programs. ${ }^{1}$ The involvement of governments, international health agencies and private sectors is necessary. ${ }^{1}$ However, each individual's circumstances are unique, and people prefer to have their individual needs met. Producing cost-effective education and counseling for an individual is a great challenge. We have seen the example of modern communication technologies being used to manage communicable diseases and other health monitoring and services in low- and middle-income countries. ${ }^{4,5}$ It appears appropriate to use such channels for dealing with the obesity epidemic. This review will provide a definition of mHealth; discuss Smartphone functions used in mHealth; and its current application in health services in the areas of lifestyles and obesity. A discussion of the scope of the existing evidence for the effectiveness of the different types of mHealth in the management of obesity using case studies is provided later in the paper. This is not intended to be an exhaustive compilation of every individual study, as systematic reviews have been published by others already. ${ }^{6-10}$

\section{A definition of mHealth}

Electronic health is described as the use of modern information and communication technology to support health care services and health surveillance. ${ }^{11}$ Mobile health, known as mHealth, is a rapidly growing part of electronic health. It is health service that is delivered using mobile and wireless devices to improve medical and public health outcomes. ${ }^{12}$ Examples include Smartphones and other mobile phones and devices such as Smart watches and wrist bands for tracking physical activity.

The 2016 survey by the Pew Global Research Centre indicated that $43 \%$ of the world's population owns a Smartphone device. ${ }^{13}$ In many countries where the prevalence of overweight and obesity is highest, the ownership of Smartphones is also more prolific and continually growing, for example, the ownership is $77 \%$ in Australia, $72 \%$ in the US and $68 \%$ in the UK. Thus, the extensive reach of Smartphones highlights their potential for widespread delivery of health services.

Different features of a Smartphone can be used to deliver obesity and healthy lifestyle management.
Smartphone-delivered management programs may be developed for independent delivery or in combination with other strategies. The functions employed to date include messaging services and multimedia messaging; Smartphone applications for lifestyle management known as "apps"; the telephone features, including voice and "FaceTime", that allow health professionals and other counselors to advise and support individuals one-on-one. Access to functions such as email and informational websites are also useful in the provision of mHealth.

The central behaviors of obesity management are diet, physical activity and sleep with these featured in many commercial Smartphone products. Smartphones and Smart watches come installed with accelerometers and algorithms to track physical activity, detect sitting, standing, walking, step counts and estimation of energy expenditure. ${ }^{14}$ These technologies are progressively being validated for their accuracy by researchers. ${ }^{15,16}$ As the role of sleep in obesity development is increasingly being researched, functions for sleep monitoring are inbuilt into wrist-worn devices. ${ }^{17,18}$ Eating habits remain the most difficult to monitor ubiquitously and still require user input in most cases. There are numerous calorie counting apps that require users to enter their food consumption. Most use the United States Department of Agriculture's food database, with additional crowdsourcing of supermarket data on products and nutritional composition. ${ }^{19,20}$ Despite the extensive food database, users from other countries may find it more difficult to locate matches to their foods. For more than a decade, the potential for digital recognition of food intake using mobile phone cameras has been investigated..$^{21,22}$ This remains problematic because of the difficulty of discerning the serving sizes (even with fiducial markers), and the composition of mixed meals has not been readily solved. The use of continuous digital photography has also been investigated using wearable cameras, but there are issues related to the positioning of the camera, ability to detect all food consumed and identification of foods. ${ }^{23}$ Self-monitoring apps and devices that measure both diet and physical activity behaviors enable individuals to become aware of their practices and may provide feedback as individuals set out to improve their health. ${ }^{19,24,25}$ However, the accuracy of all these apps and devices requires further independent evaluation and publication in peer-reviewed literature by researchers.

\section{The validation of $\mathrm{mHealth}$ for obesity management}

Private ventures, health organizations and governments all provide mHealth for obesity management. The predominant 
utility is the app, with the majority offered by the private sector having little input from health professionals with the expert knowledge and skills to manage overweight and obese individuals. ${ }^{26}$ The frameworks that are employed in testing mHealth platforms for obesity in the commercial industry sector likely differ from that in the research and clinical settings. Companies do not usually publish their processes in peer-reviewed open access forums as health researchers do. For the funders of health services, any financial inputs into mHealth for obesity need to demonstrate cost-effectiveness, as well as effectiveness and reach to the populations most at risk, in an equitable manner. ${ }^{27}$ Conversely, for commercial providers, success of the program is more likely to be judged by uptake in the market rather than successful weight loss by the majority of users. Individual testimonials are powerful tools, whereas for gains in health, the device needs to work in a large number of people most of the time. Testing usability of mHealth platforms with consumers is almost universal practice for those in the business of developing programs, but studying the effectiveness and cost-effectiveness of apps and other multicomponent programs in cohort or controlled trials is rare. ${ }^{28}$

In the following sections, we discuss the validity of three common types of mHealth programs for weight management, i.e., text messaging, apps and programs using multiple components including Smartphones.

\section{Interventions for weight management using text messaging}

A systematic review of studies using SMS indicated that 6 of 13 studies resulted in weight loss, with the duration of studies ranging from 1 to 24 months. ${ }^{6}$ A meta-regression of six of these short-term studies using text messaging for weight management, including a nutrition component indicated a mean weight loss of $2.17 \mathrm{~kg}(95 \% \mathrm{CI}-3.41$ to $-0.93 ; P<0.001)$, compared with control groups. This is similar to the mean weight loss observed in interventions using traditional delivery, such as lifestyle counseling face-to-face or groups or pharmacological agents. ${ }^{29,30} \mathrm{~A}$ previous systematic review of text messaging for weight loss that additionally included interventions without nutrition components reported that 11 of 14 interventions resulted in significant changes in weight loss or variables related to weight loss (diet and physical activity), although only 5 of 10 measuring weight demonstrated weight loss. ${ }^{31}$ Most studies included in these reviews ran for 3 months or less, so long-term effectiveness is yet to be demonstrated. A more recent trial in obese Korean men that ran for 6 months found no difference between intervention and control as both groups lost weight. ${ }^{32}$ A 2015 review of the use of text messaging to manage obesity in adolescents reported that although this medium was feasible and well accepted, its effects on changes in body mass index could not be determined from the small body of literature. ${ }^{33}$

In a small study of people signing up to a healthy eating automated texting intervention, Coa and Patrick ${ }^{34}$ reported on the high drop-out rate that occurred, finding that 83 of 193 people joining dropped out of the intervention, with 54 people discontinuing within 2 weeks. The reasons why some text message interventions fail while others succeed is likely due to the design of the program itself and the degree of tailoring to meet individual needs. Text messaging programs do not rely on the delivery medium itself for success but rather the message content and timeliness. Understanding what message content and style appeals to an individual and will enable behavior change is important to the success of these programs. The skills and knowledge, opportunities, support and individual motivations must be acknowledged and incorporated into text messages to support change in lifestyle behaviors and subsequent weight loss.

\section{Interventions for weight management using apps}

When it comes to the use of apps as the sole delivery medium for a weight loss intervention, the hyperbole exceeds the reality. Nikolaou and Lean $^{26}$ have systematically searched five different app stores in 10 countries, many with high prevalence of obesity including the UK, US, Australia and Russia. They identified 28,905 unique apps but only $0.05 \%$ indicated that they were developed with health professional input. To date, there have been a limited number of interventions using weight loss apps whether commercial or those designed for purpose by researchers. Most of these studies have been of short duration and significantly less than 2 years suggested as necessary to determine effective maintenance of weight loss. ${ }^{8}$

We previously examined the quality of 28 popular apps downloaded as part of the top 200 health and fitness apps from the Australian Google Play and iTunes stores. ${ }^{19}$ Five factors were evaluated to determine quality, including the credibility of the app developers, the accuracy and coverage of scientific information, the inclusion of technology-enhanced features to support the use of the app (e.g., barcode scanners), usability and their likelihood in changing health behaviors, assessed using Abraham and Michie's Behavior Change Taxonomy. ${ }^{35}$ Most apps had functions for entering personal data about food intake to determine energy requirements 
and for calorie counting, but compared against a food record analyzed using the Australian program based on the national Food Composition tables, there were discrepancies in energy values. There was an absence of features that would facilitate behavior change, as has also been noted by others. ${ }^{10,36,37}$ Among the most popular apps were Noom Weight ${\text { Loss } \text { Coach }^{\circledR} \text { and MyFitnessPal }}^{\circledR}$. Both these apps have been studied by researchers. ${ }^{38,39}$

There have been two approaches to examining the effectiveness of weight loss apps. One is the traditional randomized controlled trial, which is generally considered to provide best level of evidence, although a limited number of these have been conducted. ${ }^{8,10}$ The commercial app MyFitnessPal ${ }^{\circledR}$ was used in a randomized controlled trial to test its efficacy when introduced in the primary care setting in the US. After 6 months, there was no significant change in weight in those who received the app and those who did not. ${ }^{39}$ TurnerMcGrievy and Tate ${ }^{40}$ included FatSecret's Calorie Counter ${ }^{\circledR}$ app (FatSecret.com) in a randomized controlled trial and compared receiving two podcasts on weight reduction per week and an app, to a group only receiving podcasts, but reported no additional benefits for weight loss. Stephens and Allen ${ }^{41}$ added the Lose it! ${ }^{\circledR}$ app to a four-arm multicomponent intervention and also failed to find any difference in weight loss between the two arms with self-monitoring and those without, but the study was underpowered to detect this as it was a pilot study.

The results from carefully controlled trials of the use of apps designed by health professional researchers are equivocal. Carter et $\mathrm{al}^{42}$ designed My Meal Mate, and a pilot randomized controlled trial showed that those using the app lost significantly more weight at 6 months than those recording on a website or using a pen and paper diary. It is noted that using the app increased adherence to the self-monitoring regime and resulted in more study completers. Svetkey et $\mathrm{al}^{43}$ compared the use of a weight loss management app designed by health professionals alone and in combination with health coaching versus control group. No significant difference was found in weight loss between the standalone app condition and the control group; in fact, participants receiving the intervention only through the app lost the least amount of weight. The health coaching and app for self-monitoring group lost significantly more weight at 6 months than the controls, but these effects were not sustained at 12 and 24 months. Their findings highlight that health coaching and counseling may need to be a part of interventions using mHealth technologies.

More recently, there is a second approach, as some of the commercial enterprises who offer apps for weight manage- ment have made their data available to researchers. ${ }^{38,44}$ Chin et $\mathrm{al}^{38}$ analyzed the data of 35,921 participants who signed up Noom Coach $^{\circledR}$ between October 2012 and April 2014. All app users who had recorded their data two or more times for 6 months were included in the analysis. Self-reported mean baseline BMI was $30.2 \mathrm{~kg} / \mathrm{m}^{2}$ (SE 0.1 ) for men and $28.0 \mathrm{~kg} / \mathrm{m}^{2}$ (SE 0.0) for women. Participants decreased their BMIs to a mean of $28.1 \mathrm{~kg} / \mathrm{m}^{2}$ (SE 0.1 ) for men and $26.5 \mathrm{~kg} / \mathrm{m}^{2}(0.0)$ for women, with the median duration of usage of 267 days (interquartile range $=182$ days) ${ }^{38}$

The Lose It! ${ }^{\circledR}$ commercial app (Fitnow Inc., Boston, MA, USA) is very popular in the US, and the company provided data to researchers at the National Cancer Institute Bethesda. Users enter details of their weight, height, exercise level and desired weight loss and a weekly goal. The app provides them with a plan based on a daily calorie deficit to produce the desired weight loss. Data from 12,363,555 individuals were available for analysis, and researcher identified three subgroups according to the number of days they enter the data, "occasional", "basic" and "power" users. ${ }^{44}$ Those entering data for more than 40 days were more likely $(72.70 \%)$ to lose $5 \%$ of their body weight versus occasional $(4.87 \%)$ and basic users (37.61\%). ${ }^{44}$ This implies the importance of motivation to ensure successful weight loss and that frequent self-monitoring is essential. Self-monitoring builds psychological capacity for self-regulation and awareness of their behaviors and positive changes build confidence in their ability to lose weight. An analysis of behavior change techniques mediating weight loss has demonstrated the essential role self-monitoring plays. ${ }^{45}$

\section{Interventions using multiple mHealth components}

mHealth interventions often utilize a combination of Smartphone delivery functions. Thomas and Wing ${ }^{46}$ reported the utility of using a Smartphone to deliver intervention components in a multicomponent program. The intervention consisted of Smartphone self-monitoring, automated and human feedback, and education and skills training via video, as well as weekly in-person weigh-ins and paper handouts, which were found to produce an average of $9 \%$ loss of initial body weight $(8.4 \mathrm{~kg})$ at 12 weeks and $11 \%$ $(10.9 \mathrm{~kg})$ at 24 weeks. These findings indicate that Smartphones could be mediums for enhancing the delivery of personalized behavioral weight loss treatments, thereby reducing cost and resources required for face-to-face treatments. A randomized controlled trial of a multicomponent intervention to prevent weight gain in younger adults that 
included brief coaching calls, text messaging, emails, apps and downloadable resources resulted in a significant decrease in weight in the intervention arm versus control at 12 weeks and even greater differences at 9 months. ${ }^{47}$ Godino et $\mathrm{al}^{48}$ also tested a multicomponent intervention for young adults using social and mobile media in a 24-month-long randomized controlled trial, but while the intervention group had lost significantly more weight at 6 months, this was not maintained at 24 months.

\section{Adaptive mHealth interventions}

An important feature for any "Smart" app or device designed to assist weight loss is that the user will engage with the app sufficiently to receive the "treatment". The app or device likely needs to include some of the behavior change techniques demonstrated to alter eating and physical activity behaviors. ${ }^{49}$ Timeliness of intervention messages is also important, such that they prompt or cue a behavior appropriately, for example, reminders to go for that early morning walk, avoid the alcohol binge on Friday nights or to add some vegetables at lunch time. To date, most interventions with mHealth rely on the individual making a conscious effort to modify their behavior at times of temptation to revert to the behavior they are attempting to avoid. However, as remote sensing evolves it should be possible for the Smartphone to deliver help at just the right time. This leads to the concept of "Just-in-Time-Adaptive" interventions. ${ }^{50-52}$

The ability to assess behaviors in real time using Smart devices provides the potential to continuously adapt interventions according to an individual's behavioral response at the last treatment point. While it is common practice to tailor interventions based on the reported baseline characteristics of the individual, we should now be able to find the means to alter interventions continuously according to how the individual's measured behavior is changing. However, we are only just beginning to understand how to optimize such interventions. ${ }^{53-55}$ The behavioral response to a given intervention is based on real-time sensing, for example, physical activity and sitting time and real-time input of data by an individual, such as recording food intake prospectively throughout the day. Using intensive adaptive interventions raises new questions and decision-making, such as how frequently an intervention should be adapted in response to a single measured output and whether the perceived intrusive nature of such an intervention will mean people will abandon their intervention before new eating habits and physical activity give rise to weight loss. ${ }^{53}$ The technical challenges in monitoring dietary intake without any input from the individual means that progress in adapting interventions for changing eating behaviors is more restricted than physical activity, where sensing can be done passively and unconsciously on the individual's part.

\section{Consumer opinions of using mHealth for weight management}

The consumers who will use an app should always be central to the design of any mHealth program. An important consideration in the development of mHealth devices and programs for managing the behaviors that determine successful weight management is the beliefs and needs of those for whom it is designed, i.e., individuals with overweight and obesity. Much of the research conducted to date has been centered on the physical outcomes of change in body weight or change in eating and physical activity behaviors. However, studying consumer opinions on the process itself is required to give insight into how to make mHealth technologies and interventions better for the end user. The findings from the available research have similarities to that from earlier forms of weight loss programs. Personalization of material is important for continued engagement and behavior changes. The learnings about user preferences for apps were not dissimilar to consumer preferences for any Smartphone apps; visually appealing, easy to navigate and feedback is relevant. However, there are some preferences that are distinctive for mHealth. ${ }^{56}$ Participants in two different Australian studies indicated that they preferred intervention components be delivered via a single app on the Smartphone without the need to ever use the web on a computer. ${ }^{57,58}$ Also suggested was the incorporation of feedback into the app that is individualized and features visual elements and the inclusion of rewards systems when progress is made (Nour M, et al, University of Sydney, unpublished data, 2017). ${ }^{59}$

More is known about consumer preferences for text messaging interventions. ${ }^{56}$ In this case, the personalization desired is with respect to content relevance, timeliness of the message, tone of the messages and frequency of messaging. There is no overarching advice as to what ensures a successful text messaging program for overweight and obesity but consideration of the aforementioned elements in the target group for a given intervention is required. Messages will need to vary depending on age, socioeconomic status, literacy, knowledge and skills, readiness for change and personal motivations. Any mHealth weight management intervention must be matched to the needs of the target group and one size does not fit all. 


\section{Conclusion}

The current pandemic of obesity dictates the urgency of deployable solutions for the global population. The use of Smart technologies for mHealth in weight management is one of the numerous possibilities being explored. There are hundreds of thousands of health and lifestyle apps but the most popular for weight management are those that selfmonitor calorie intake and physical activity. These should be combined with goal setting and feedback on goals to enhance the likelihood of weight management. All individuals who struggle to reach and maintain a healthy weight have unique circumstances, and in an ideal world would receive individual care with a qualified health professional, but the scope of the problem is so large that this is impossible. Technology offers the promise of being able to simulate individual treatment as we advance the capabilities to adjust mHealth depending on outputs from the consumer and adjustment of advice in real time to meet their needs. However, it must be remembered that this is only one part of the solution and other parts, such as policies to make food environments healthier, require similar continuing investments. There is clearly a need for behavioral and clinical scientists to work with the computational modelers, information technologists and engineers to develop Smartphone apps and devices together for maximum benefit to the overweight and obese population.

\section{Disclosure}

MAF has designed nutrition apps for use in research but this review has not received any funding. She has previously had research funding from the HCF Medical Research Foundation and currently has funding from the Australian Research Council. JC is supported by a $\mathrm{PhD}$ scholarship from the Australian Research Council. The authors report no other conflicts of interest in this work.

\section{References}

1. World Health Organisation. Obesity and Overweight Factsheet; 2016. Available from: http:/www.who.int/mediacentre/factsheets/fs311/en/. Accessed February 17, 2017.

2. World Cancer Research Fund/American Institute for Cancer Research. Food, Nutrition, Physical Activity, and the Prevention of Cancer: a Global Perspective; 2007. Available from: http:/www.aicr.org/assets/ docs/pdf/reports/Second_Expert_Report.pdf. Accessed February 17, 2017.

3. GBD 2015 Risk Factors Collaborators. Global, regional, and national comparative risk assessment of 79 behavioural, environmental and occupational, and metabolic risks or clusters of risks, 1990-2015: a systematic analysis for the Global Burden of Disease Study 2015. Lancet. 2016;388(10053):1659-1724.

4. Bastawrous A, Armstrong MJ. Mobile health use in low- and highincome countries: an overview of the peer-reviewed literature. $J R$ Soc Med. 2013;106(4):130-142.
5. Agarwal S, Perry HB, Long LA, Labrique AB. Evidence on feasibility and effective use of mHealth strategies by frontline health workers in developing countries: systematic review. Trop Med Int Health. 2015;20(8): 1003-1014.

6. Siopis G, Chey T, Allman-Farinelli M. A systematic review and metaanalysis of interventions for weight management using text messaging. J Hum Nutr Diet. 2015;28(suppl 2):1-15.

7. DiFilippo KN, Huang WH, Andrade JE, Chapman-Novakofski KM. The use of mobile apps to improve nutrition outcomes: a systematic literature review. J Telemed Telecare. 2015;21(5):243-253.

8. Aguilar-Martínez A, Solé-Sedeño JM, Mancebo-Moreno G, Medina FX, Carreras-Collado R, Saigí-Rubió F. Use of mobile phones as a tool for weight loss: a systematic review. J Telemed Telecare. 2014;20(6): 339-349.

9. Bardus M, Smith JR, Samaha L, Abraham C. Mobile phone and web 2.0 technologies for weight management: a systematic scoping review. J Med Internet Res. 2015;17(11):e259.

10. Liu F, Kong X, Cao J, et al. Mobile phone intervention and weight loss among overweight and obese adults: a meta-analysis of randomized controlled trials. Am J Epidemiol. 2015;181(5):337-348.

11. World Health Organisation [webpage on the Internet]. eHealth. Available from: http://www.who.int/topics/ehealth/en/. Accessed February $17,2017$.

12. World Health Organisation. mHealth: New Horizons for Health Through Mobile Technologies: Second Global Survey on eHealth; 2011. Available from: http://www.who.int/goe/publications/goe_mhealth_web.pdf. Accessed December 10, 2015.

13. Poushter J. Smartphone Ownership and Internet Usage Continues to Climb in Emerging Economies; 2016. Available from: http://www. diapoimansi.gr/PDF/pew_research\%201.pdf. Accessed June 13, 2017.

14. Guiry JJ, van de Ven P, Nelson J, Warmerdam L, Riper H. Activity recognition with smartphone support. Med Eng Phys. 2014;36(6): 670-675.

15. Hekler EB, Buman MP, Grieco L, et al. Validation of physical activity tracking via android smartphones compared to ActiGraph accelerometer: laboratory-based and free-living validation studies. JMIR Mhealth Uhealth. 2015;3(2):e36.

16. Tucker WJ, Bhammar DM, Sawyer BJ, Buman MP, Gaesser GA. Validity and reliability of Nike + Fuelband for estimating physical activity energy expenditure. BMC Sports Sci Med Rehabil. 2015;7:14.

17. Lee PH, Suen LK. The convergent validity of Actiwatch 2 and ActiGraph Link accelerometers in measuring total sleeping period, wake after sleep onset, and sleep efficiency in free-living condition. Sleep Breath. 2017;21(1):209-215.

18. Lee J, Finkelstein J. Consumer sleep tracking devices: a critical review. Stud Health Technol Inform. 2015;210:458-460.

19. Chen J, Cade JE, Allman-Farinelli M. The most popular smartphone apps for weight loss: a quality assessment. JMIR MHealth UHealth. 2015;3(4):e104.

20. Gan KO, Allman-Farinelli M. A scientific audit of smartphone applications for the management of obesity. Aust $N$ Z J Public Health. 2011;35(3):293-294.

21. Boushey CJ, Spoden M, Zhu FM, Delp EJ, Kerr DA. New mobile methods for dietary assessment: review of image-assisted and image-based dietary assessment methods. Proc Nutr Soc. Epub 2016 Dec 12:1-12.

22. Gemming L, Utter J, Ni Mhurchu C. Image-assisted dietary assessment: a systematic review of the evidence. J Acad Nutr Diet. 2015;115(1): 64-77.

23. Farinella GM, Allegra D, Moltisanti M, Stanco F, Battiato S. Retrieval and classification of food images. Comput Biol Med. 2016;77:23-39.

24. Orr K, Howe HS, Omran J, et al. Validity of smartphone pedometer applications. BMC Res Notes. 2015;8:733.

25. Sharp DB, Allman-Farinelli M. Feasibility and validity of mobile phones to assess dietary intake. Nutrition. 2014;30(11-12):1257-1266.

26. Nikolaou CK, Lean ME. Mobile applications for obesity and weight management: current market characteristics. Int J Obes. 2017;41(1):200-202. 
27. Bergmo TS. How to measure costs and benefits of eHealth interventions: an overview of methods and frameworks. $J$ Med Internet Res. 2015;17(11):e254.

28. Boulos MNK, Brewer AC, Karimkhani C, Buller DB, Dellavalle RP. Mobile medical and health apps: state of the art, concerns, regulatory control and certification. Online J Public Health Inform. 2014;5(3):e229.

29. Franz MJ, VanWormer JJ, Crain AL, et al. Weight-loss outcomes: a systematic review and meta-analysis of weight-loss clinical trials with a minimum 1-year follow-up. JAm Diet Assoc. 2007;107(10):1755-1767.

30. Neovius M, Johansson K, Rossner S. Head-to-head studies evaluating efficacy of pharmaco-therapy for obesity: a systematic review and meta-analysis. Obes Rev. 2008;9(5):420-427.

31. Shaw R, Bosworth H. Short message service (SMS) text messaging as an intervention medium for weight loss: a literature review. Health Informatics J. 2012;18(4):235-250.

32. Kim JY, Oh S, Steinhubl S, et al. Effectiveness of 6 months of tailored text message reminders for obese male participants in a worksite weight loss program: randomized controlled trial. JMIR Mhealth Uhealth. 2015;3(1):e14 .

33. Keating SR, McCurry MK. Systematic review of text messaging as an intervention for adolescent obesity. J Am Assoc Nurse Pract. 2015;27(12):714-720.

34. Coa K, Patrick H. Baseline motivation type as a predictor of dropout in a healthy eating text messaging program. JMIR Mhealth Uhealth 2016;4(3):e114.

35. Abraham $\mathrm{C}$, Michie S. A taxonomy of behavior change techniques used in interventions. Health Psychol. 2008;27(3):379-387.

36. Direito A, Dale LP, Shields E, Dobson R, Whittaker R, Maddison R. Do physical activity and dietary smartphone applications incorporate evidence-based behaviour change techniques? BMC Public Health 2014;14(1):646.

37. Pagoto S, Schneider K, Jojic M, DeBiasse M, Mann D. Evidence-based strategies in weight-loss mobile apps. Am J Prev Med. 2013;45(5): 576-582.

38. Chin SO, Keum C, Woo J, et al. Successful weight reduction and maintenance by using a smartphone application in those with overweight and obesity. Sci Rep. 2016;6:34563.

39. Laing BY, Mangione CM, Tseng C-H, et al. Effectiveness of a smartphone application for weight loss compared with usual care in overweight primary care patients: a randomized, controlled trial. Ann Intern Med. 2014;161(10_Supplement):S5-S12.

40. Turner-McGrievy G, Tate D. Tweets, Apps, and Pods: results of the 6-month mobile pounds off digitally (Mobile POD) randomized weightloss intervention among adults. J Med Internet Res. 2011;13(4):e120.

41. Stephens J, Allen J. Mobile phone interventions to increase physical activity and reduce weight: a systematic review. J Cardiovasc Nurs. 2013;28(4):320-329.

42. Carter MC, Burley VJ, Nykjaer C, Cade JE. Adherence to a smartphone application for weight loss compared to website and paper diary: Pilot randomized controlled trial. J Med Internet Res. 2013;15(4):e32.

43. Svetkey LP, Batch BC, Lin PH, et al. Cell phone intervention for you (CITY): a randomized, controlled trial of behavioral weight loss intervention for young adults using mobile technology. Obesity (Silver Spring). 2015;23(11):2133-2141.
44. Serrano KJ, Yu M, Coa KI, Collins LM, Atienza AA. Mining health app data to find more and less successful weight loss subgroups. J Med Internet Res. 2016;18(6):e154.

45. Michie S, Abraham C, Whittington C, McAteer J, Gupta S. Effective techniques in healthy eating and physical activity interventions: a metaregression. Health Psychol. 2009;28(6):690-701.

46. Thomas JG, Wing RR. Health-e-call, a smartphone-assisted behavioral obesity treatment: pilot study. JMIR Mhealth Uhealth. 2013;1(1):e3.

47. Allman-Farinelli M, Partridge SR, McGeechan K, et al. A mobile health lifestyle program for prevention of weight gain in young adults (TXT2BFiT): nine-month outcomes of a randomized controlled trial. JMIR Mhealth Uhealth. 2016;4(2):e78.

48. Godino JG, Merchant G, Norman GJ, et al. Using social and mobile tools for weight loss in overweight and obese young adults (Project SMART): a 2 year, parallel-group, randomised, controlled trial. Lancet Diabetes Endocrinol. 2016;4(9):747-755.

49. Bardus M, van Beurden SB, Smith JR, Abraham C. A review and content analysis of engagement, functionality, aesthetics, information quality, and change techniques in the most popular commercial apps for weight management. Int J Behav Nutr Phys Act. 2016;13:35.

50. Intille SS, Larson K. Designing and evaluating home-based, just-intime supportive technology. Stud Health Technol Inform. 2005;118: 79-88.

51. Patrick K, Griswold WG, Raab F, Intille SS. Health and the mobile phone. Am J Prev Med. 2008;35(2):177-181.

52. Nahum-Shani I, Hekler EB, Spruijt-Metz D. Building health behavior models to guide the development of just-in-time adaptive interventions: a pragmatic framework. Health Psychol. 2015;34s:1209-1219.

53. Riley WT, Serrano KJ, Nilsen W, Atienza AA. Mobile and wireless technologies in health behavior and the potential for intensively adaptive interventions. Curr Opin Psychol. 2015;5:67-71.

54. Klasnja P, Hekler EB, Shiffman S, et al. Microrandomized trials: an experimental design for developing just-in-time adaptive interventions. Health Psychol. 2015;34s:1220-1228.

55. Thomas JG, Bond DS. Behavioral response to a just-in-time adaptive intervention (JITAI) to reduce sedentary behavior in obese adults: implications for JITAI optimization. Health Psychol. 2015;34s: 1261-1267.

56. Lyzwinski LN, Caffery LJ, Bambling M, Edirippulige S. Consumer perspectives on mHealth for weight loss: a review of qualitative studies J Telemed Telecare. Epub 2017 Jan 1.

57. Partridge SR, Allman-Farinelli M, McGeechan K, et al. Process evaluation of TXT2BFiT: a multi-component $\mathrm{mHealth}$ randomised controlled trial to prevent weight gain in young adults. Int J Behav Nutr Phys Act. 2016;13:7.

58. Vandelanotte C, Caperchione CM, Ellison M, et al. What kinds of website and mobile phone-delivered physical activity and nutrition interventions do middle-aged men want? J Health Commun. 2013;18(9):1070-1083.

59. Tang J, Abraham C, Stamp E, Greaves C. How can weight-loss app designers' best engage and support users? A qualitative investigation. Br J Health Psychol. 2015;20(1):151-171.
Smart Homecare Technology and TeleHealth

\section{Publish your work in this journal}

Smart Homecare Technology and TeleHealth is an international, peer-reviewed, open access online journal publishing original research, reviews, editorials and commentaries on the application of technology to support people and patients at home and in assisted living centers to optimize healthcare and management resources. Specific topics in the journal include: Development and application of
Dovepress

devices within the home and embedded in appliances; Healthcare provider communication and education tools; and drug ordering and adherence. The manuscript management system is completely online and includes a very quick and fair peer-review system, which is all easy to use. Visit http://www.dovepress.com/ testimonials.php to read real quotes from published authors. 